\title{
Non-collisional cross-field diffusion of relativistic electrons
}

\author{
E. Amato, M. Pettini, and M. Salvati
}

INAF/Osservatorio Astrofisico di Arcetri, Largo E. Fermi 5, 50125 Firenze, Italy

Received 14 May 2002 / Accepted 7 February 2003

\begin{abstract}
We investigate the diffusion of relativistic particles in the presence of a time constant, spatially non-homogeneous magnetic field. We show that the intrinsic dynamical instability of the particle trajectories can provide an important source of non-collisional diffusion even in the absence of time-dependent phenomena and for the simplest possible field structure that still allows microscopic chaos.
\end{abstract}

Key words. relativity - instabilities - magnetic fields - plasmas - shock waves

\section{Introduction}

Cross field particle diffusion is likely to play an important role in magnetized plasmas every time one deals with energetic particles with large Larmor radii. Of course this happens in a number of different astrophysical situations and in particular in the context of high frequency radiating synchrotron sources.

The question of what the efficiency of diffusion is has considerable importance both for the understanding of particle transport and for the problems relating to particle acceleration at shocks. Coulomb collisions are essentially absent in astrophysical plasmas and the source of efficient diffusion is usually looked for in magnetic field irregularities that produce wandering of the field lines themselves or in the scattering of particles with waves (e.g. Melrose 1980). In particular, a widespread point of view about diffusion of charged particles attributes it to the particle dragging by sticky magnetic lines that chaotically wander in space. A wealth of literature is available on this subject and on its applications to a variety of astrophysical environments. Let us just quote a few of them because of their relevance to our present work: recent detailed numerical studies (Casse et al. 2001) provide highly refined information about parallel and transverse spatial diffusion coefficients in the presence of 3D magnetic turbulence, whereas theoretical results (Jones et al. 1998; Jokipii et al. 1993) somewhat constrain the magnetic field structure in order to allow cross-field diffusion.

However, even in the absence of Coulomb collisions, crossfield Brownian-like diffusion can be induced by chaotic particle dynamics. Chaotic particle motions are commonly found in presence of inhomogeneous electric fields (typically of interest to fusion plasmas, see for example Pettini et al. 1988; Ottaviani \& Pettini 1989, 1991), or in presence of inhomogeneous magnetic fields, in which case the simplest example is provided by a charged particle moving in a magnetic dipole

Send offprint requests to: E. Amato, e-mail: amato@arcetri.astro.it field (De Alcantara Bonfim et al. 2000). Thus, turbulent magnetic fields are not absolutely necessary for particle diffusion, "mild" inhomogeneities can be effective as well.

The aim of our present work is to draw attention to this other physical mechanism which can either give rise to crossfield diffusion by itself, or can cooperate, and perhaps even compete, with the stochastic wandering of magnetic field lines. This consists of intrinsic dynamical chaos directly arising from the microscopic equations of motion when the magnetic field is not perfectly uniform or does not fulfil suitable symmetry requirements (Jokipii et al. 1993). To this aim, we considered the simplest possible configuration (meaning the one with the minimum number of degrees of freedom) still guarantees a degree of complexity sufficient to entail diffusion. We remark that the model considered throughout this paper is the only one which is non-trivial in the light of a theoretical result ruling out diffusion in the case of magnetic fields with one ignorable spatial coordinate (Jones et al. 1998). Moreover, at variance with what has been found in Casse et al. (2001), in our model Bohm scaling is not ruled out, therefore we might venture the guess that a non-trivial interplay between chaotic motions of particles and chaotic wandering of field lines could be responsible for the appearance of unexpected results.

\section{Outline of the model}

We took the magnetic field to be directed along the $z$ axis in a Cartesian system of coordinates and considered the particles motion in the $(x, y)$ plane. The magnetic field was taken to be in the form:

$\boldsymbol{B}=B_{0}\left(1+\sum_{i=1}^{n} A_{\boldsymbol{k}^{i}} \cos \left(k_{x}{ }^{i} x+k_{y}{ }^{i} y+\phi^{i}\right)\right) \hat{\boldsymbol{k}}$

with $\left|\boldsymbol{k}^{i}\right|=2 \pi / \lambda^{i}$, i.e. a constant uniform magnetic field $B_{0} \hat{\boldsymbol{k}}$ with a superposed stationary and spatially inhomogeneous component $B_{1}(x, y) \hat{\boldsymbol{k}}$, here represented as a finite sum of Fourier components. 
Particles are only subject to the Lorentz force, so that the equation of motion reads:

$$
\frac{\mathrm{d} \boldsymbol{\beta}}{\mathrm{d} t}=\frac{e}{m \gamma c}(\boldsymbol{\beta} \wedge \boldsymbol{B})
$$

with $e, m$ and $\gamma$ being the particle charge, mass and Lorentz factor respectively, $c$ representing the speed of light and $\beta=v / c$, where $\boldsymbol{v}$ is the particle velocity.

This second order vector differential equation translates into a system of four first order scalar equations. But these are not all independent because of the constraint provided by energy conservation, appropriate to the situation we are dealing with as long as radiation losses can be ignored. For time scales short in comparison with the particle synchrotron lifetimes, $\beta$ is a constant and we can reduce the system to only three scalar equations, writing:

$$
\begin{aligned}
\frac{1}{c} \frac{\mathrm{d} x}{\mathrm{~d} t} & =\beta_{x}=\beta \cos \alpha \\
\frac{1}{c} \frac{\mathrm{d} y}{\mathrm{~d} t} & =\beta_{y}=\beta \sin \alpha \\
\frac{\mathrm{d} \alpha}{\mathrm{d} t} & =-\frac{e B(x, y)}{m \gamma c}
\end{aligned}
$$

where $B(x, y)$ is given by Eq. (1) with a suitable choice of the amplitude coefficients $A_{\boldsymbol{k}^{i}}$ and of the phases $\phi^{i}$.

The equations of motion (3) are nonlinear and, with the exception of a few very special cases, no general method for their analytic solution is available. A numeric study is therefore necessary to work out the particle trajectories and estimate the effects of diffusion if a diffusive behaviour is found.

\section{Numerical investigation of particles diffusion in a magnetic field with fluctuating strength}

We performed a numerical study aimed at clarifying which, if any, are the magnetic field configurations effective for producing cross field particles diffusion within the framework of the model outlined in the previous section.

The parameters that are expected to be relevant in determining the efficiency of particle diffusion are the perturbation amplitude and spectrum. We varied them both in our simulations, as we specify below.

\subsection{Magnetic field description}

We took the wavenumbers $\boldsymbol{k}^{i}$ in the form:

$$
\left\{\begin{array}{l}
k_{x}{ }^{i}=\frac{2 \pi}{\lambda_{\mathrm{M}}} n_{i} \\
k_{y}{ }^{i}=\frac{2 \pi}{\lambda_{\mathrm{M}}} m_{i}
\end{array} \text { with } 1<n_{i}{ }^{2}+m_{i}{ }^{2}<\rho^{2},\right.
$$

where $\rho=\lambda_{\mathrm{M}} / \lambda_{\mathrm{m}}$ and $\lambda_{\mathrm{M}}$ and $\lambda_{\mathrm{m}}$ are, respectively, the maximum and minimum perturbation wavelength taken into account. The latter vary among the different simulations and so does the spectral form of the perturbation. In particular, as far as the power spectrum of the perturbation is concerned we have considered both a flat spectrum $A_{\boldsymbol{k}^{i}}=A_{1}$, with $A_{1}$ a constant, i.e. a field configuration such that more power is in short wavelength modes, and a power-law spectrum of index -1 ,
$A_{\boldsymbol{k}^{i}}=A_{2} k^{i^{-1}}$, which in 2D is equivalent to evenly distributing the total energy in the perturbation among the different spatial scales. As to $A_{1}$ and $A_{2}$ these were determined through the condition:

$\frac{1}{2} \sum_{i=1}^{n} A_{\boldsymbol{k}^{i}}^{2}=\left(\frac{\delta B}{B_{0}}\right)^{2}$,

with $\delta B_{0} / B_{0}$, once again, varying.

Finally, the phases $\phi^{i}$ were chosen randomly.

\subsection{The relevant observables}

There are two observables of central interest to our study: the mean square displacement as a function of time, and the Lyapunov characteristic exponent. These two observables allow to compute the diffusion coefficient, and to test whether the dynamics is unstable (chaotic), respectively.

By numerically following the trajectories of $N$ particles, and denoting by $\boldsymbol{x}_{i}(0), i=1, \ldots, N$ their initial conditions and by $\boldsymbol{x}_{i}(t), i=1, \ldots, N$ their respective positions at any time $t$, the mean square displacement is simply obtained as

$\left\langle r^{2}(t)\right\rangle=\frac{1}{N} \sum_{i=1}^{N}\left|\boldsymbol{x}_{i}(t)-\boldsymbol{x}_{i}(0)\right|^{2}$.

Hence, if the asymptotic-time dependence of $\left\langle r^{2}(t)\right\rangle$ happens to be linear, it is possible to define a dynamical (non-collisional) diffusion coefficient (Lichtenberg \& Lieberman 1992) from

$D=\lim _{t \rightarrow \infty} \frac{1}{t}\left\langle r^{2}(t)\right\rangle$

For what concerns the Lyapunov characteristic exponent $\Lambda$, let us briefly spend a few words describing deterministic chaos. During the last two decades or so, there has been a growing evidence of the independence of the two properties of determinism and predictability of classical dynamics. In fact, predictability for arbitrary long times requires also the stability of the motions with respect to variations - however small - of the initial conditions.

The generic situation of classical dynamical systems described by three or more nonlinear differential equations is instability of the trajectories in the Lyapunov sense. Like any other kind of instability, dynamical instability brings about the exponential growth of an initial perturbation; in this case it is the distance between a reference trajectory and any other trajectory originating in its close vicinity that locally grows exponentially in time.

The degree of chaoticity of a dynamical system is quantified by the Lyapunov characteristic exponent $\Lambda$ which - if positive - measures the mean instability rate of nearby trajectories averaged along a sufficiently long reference trajectory. The exponent $\Lambda$ also measures the typical time scale of loss of memory of the initial conditions. Let us briefly mention how $\Lambda$ is numerically computed. To any generic dynamical system described by the equations

$\dot{x}^{i}=X^{i}\left(x^{1} \ldots x^{N}\right)$ 
we can associate the so-called tangent dynamics equations (Lichtenberg \& Lieberman 1992)

$\dot{\xi}^{i}=\sum_{k=1}^{N} \mathcal{J}_{k}^{i}[x(t)] \xi^{k} \equiv \sum_{k=1}^{N} \frac{\partial X^{i}}{\partial x^{k}} \xi^{k}$

where $\left[\mathcal{J}_{k}^{i}\right]$ is the Jacobian matrix of $\left[X^{i}\right]$, describing the evolution of the tangent vector $\xi$ which is tightly related to the local evolution of the distance of nearby trajectories to a reference trajectory.

The numerical estimate of the Lyapunov characteristic exponent is obtained, for a single trajectory, through the following formula:

$\Lambda\left(t_{\mathcal{N}}\right)=\frac{1}{t_{\mathcal{N}}} \ln \frac{\left|\boldsymbol{\xi}\left(t_{\mathcal{N}}\right)\right|}{\left|\boldsymbol{\xi}\left(t_{0}\right)\right|}$

where $t_{\mathcal{N}}$ is such that $\Lambda\left(t_{\mathcal{N}}\right)$ has reasonably attained an asymptotic value (Benettin et al. 1976). A positive asymptotic value of $\Lambda$ implies chaotic dynamics, whereas $\Lambda(t) \sim t^{-1}$ corresponds to non-chaotic dynamics.

\subsection{Simulations set-up}

In our simulations the field was computed on a grid with $N_{x} \times$ $N_{y}$ cells of size $\Delta x \times \Delta y$, each. The cells were such that $\Delta=$ $\Delta x=\Delta y=\lambda_{\mathrm{m}} / 5$ and $N_{x}=N_{y}=\lambda_{\mathrm{M}} / \Delta$. The value of the field at each point in space was found through linear interpolation using 4 neighbouring grid points.

After testing how the choice of the number of particles considered affects the results, we followed the motion of a set of $N=100$ test particles for each choice of the different parameters discussed above. The initial conditions of the different particles were chosen randomly, and together with the system of equations describing the particle dynamics also the associated one describing the tangent dynamics was integrated.

The equations were made non-dimensional by defining the set of variables:

$q_{1}=x / r_{\mathrm{L}}, \quad q_{2}=y / r_{\mathrm{L}}, \quad q_{3}=\alpha, \quad \tau=\Omega_{\mathrm{L}} t$,

with $r_{\mathrm{L}}=m \gamma c^{2} / e B_{0}$ and $\Omega_{\mathrm{L}}=c / r_{\mathrm{L}}$. Hence, the system of equations, for each choice of the parameters defining the field and for each initial condition, is:

$$
\begin{aligned}
& \frac{\mathrm{d} q_{1}}{\mathrm{~d} \tau}=\beta \cos q_{3} \\
& \frac{\mathrm{d} q_{2}}{\mathrm{~d} \tau}=\beta \sin q_{3} \\
& \frac{\mathrm{d} q_{3}}{\mathrm{~d} \tau}=-b\left(q_{1}, q_{2}\right) \\
& \frac{\mathrm{d} \xi_{1}}{\mathrm{~d} \tau}=-\beta \sin q_{3} \xi_{3} \\
& \frac{\mathrm{d} \xi_{2}}{\mathrm{~d} \tau}=\beta \cos q_{3} \xi_{3} \\
& \frac{\mathrm{d} \xi_{3}}{\mathrm{~d} \tau}=-\left(\frac{\partial b}{\partial q_{1}} \xi_{1}+\frac{\partial b}{\partial q_{2}} \xi_{2}\right)
\end{aligned}
$$

with $b\left(q_{1}, q_{2}\right)=B\left(x / r_{\mathrm{L}}, y / r_{\mathrm{L}}\right) / B_{0}$.

The integration was performed using a fourth order Milne modified predictor corrector (see e.g. Numerical Recipes).
For purposes of comparison and program testing, for some meaningful choices of the field configuration we integrated, together with the equations above, an analogous system of equations describing the guiding center motion in the gradient drift approximation. The latter are supposed to describe the particle trajectories in the case where the minimum spatial scale on which the magnetic field varies is much larger than their Larmor radius, and are found by averaging Eq. (2) over a gyration period. One obtains:

$\frac{\mathrm{d} \boldsymbol{x}_{\mathrm{gc}}}{\mathrm{d} t}=\frac{r_{\mathrm{L}} c}{2 B_{0}^{2}} \boldsymbol{\nabla} B \wedge \boldsymbol{B}$.

After rescaling space and time as in Eq. (11), we can write the system of equations relevant for cases when the guiding center approximation holds as:

$$
\begin{aligned}
\frac{\mathrm{d} q_{1}}{\mathrm{~d} \tau} & =\frac{1}{2} \frac{\partial b}{\partial q_{2}} \\
\frac{\mathrm{d} q_{2}}{\mathrm{~d} \tau} & =-\frac{1}{2} \frac{\partial b}{\partial q_{1}} \\
\frac{\mathrm{d} \xi_{1}}{\mathrm{~d} \tau} & =\frac{1}{2}\left[\frac{\partial^{2} b}{\partial q_{2} \partial q_{1}} \xi_{1}+\frac{\partial^{2} b}{\partial^{2} q_{2}} \xi_{2}\right] \\
\frac{\mathrm{d} \xi_{2}}{\mathrm{~d} \tau} & =-\frac{1}{2}\left[\frac{\partial^{2} b}{\partial^{2} q_{1}} \xi_{1}+\frac{\partial^{2} b}{\partial q_{1} \partial q_{2}} \xi_{2}\right] .
\end{aligned}
$$

We notice that the latter system of equations does not admit chaoticity, since the dynamics is described by just two independent first order differential equations. In the following we shall discuss how good an approximation the $\nabla B$ drift is in different situations and down to what values of the ratio $\lambda_{\mathrm{m}} / r_{\mathrm{L}}$ it applies.

\section{Results}

In this section we summarize our results.

First of all let us consider a situation in which the wave spectrum extends from $\lambda_{\mathrm{m}}=10 r_{\mathrm{L}}$ to $\lambda_{\mathrm{M}}=100 r_{\mathrm{L}}$ with a total of 400 wave-modes. We take the perturbation amplitude $\left(\delta B / B_{0}\right)=0.5$ (Eq. (5)) and the particle velocity to be very close to the speed of light, corresponding to $\gamma \simeq 10^{6}$. The perturbation spectrum is $\propto k^{-1}$.

Since the minimum wavelength included in the spectrum is large compared to the particles Larmor radius, this is a case such that the drift approximation is expected to give a reasonable description of most particles' trajectories. In fact, Fig. 1 shows the comparison between the motion of real particles (left panel), found through integration of Eq. (3), and of the respective drifting guiding centers (right panel), computed by integrating Eq. (13).

First of all the results in the bottom panel of Fig. 1 allow a check of the accuracy of the integration routine. One finds that the drift trajectories are actually coincident with the magnetic field iso-contours to a very good accuracy.

Furthermore, it is apparent that while for some particles the guiding center drift approximation is a good description of the real trajectories, for some others this is not the case already for a field varying over scales that are indeed large compared to $r_{\mathrm{L}}$. 

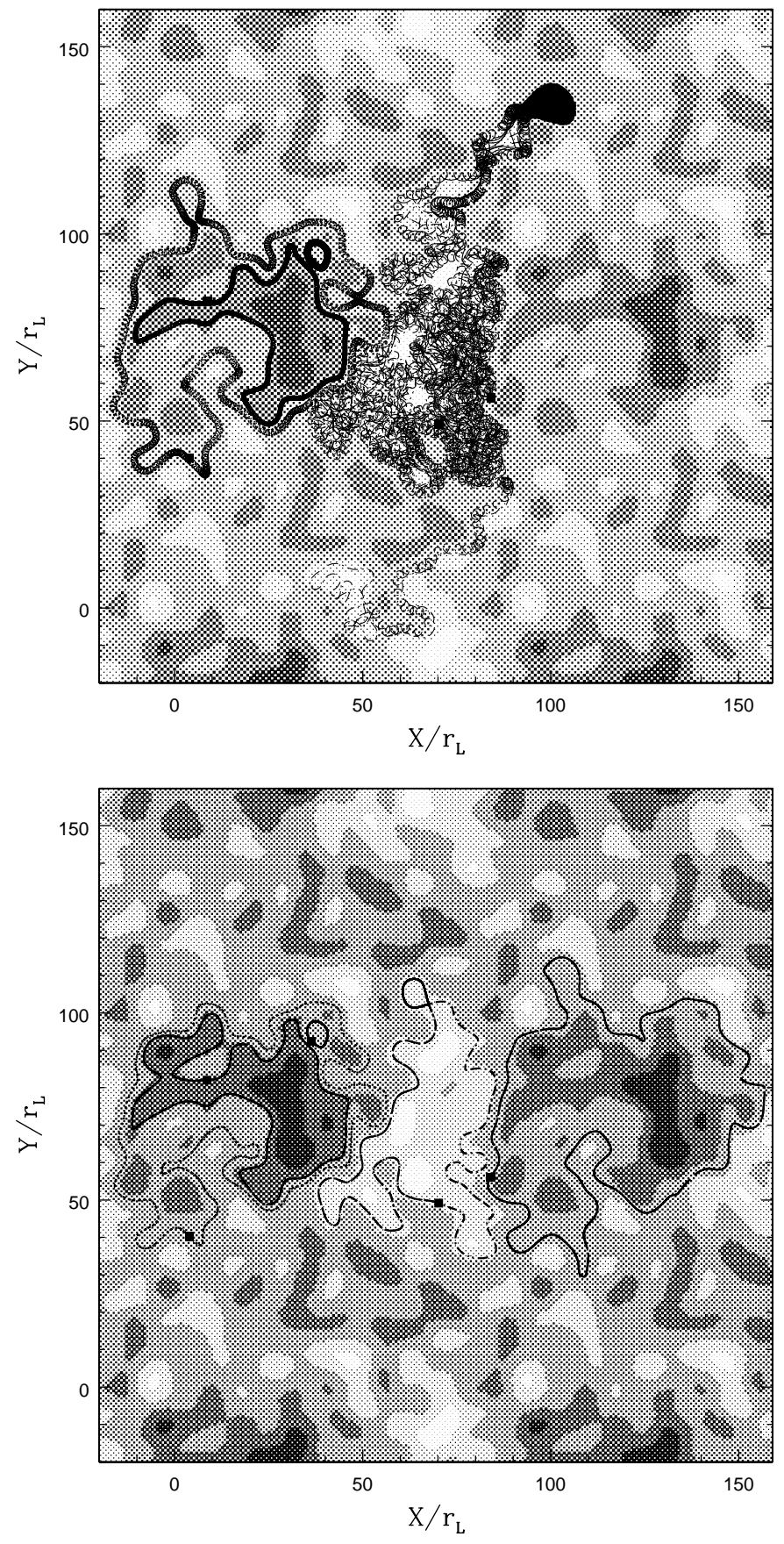

Fig. 1. The trajectories obtained by integrating the particles equation of motion (top panel) are compared with those computed integrating the gradient drift equation (bottom panel) for the same intial condition. In both cases we plot the trajectories against a grey-scale plot of the magnetic field strength.

Of course the situation becomes worse when one goes to fields varying over smaller scales.

In Fig. 2 we show a plot analogous to that in Fig. 1 but for the case when the magnetic field includes modes of wavelength between $r_{\mathrm{L}}$ and $10 r_{\mathrm{L}}$. In this case the motion of real particles has nothing to do with that expected based on a drift. Moreover, the trajectories plotted in Fig. 1 are shown after having been followed for a much longer time than those in Fig. 2. It is qualitatively apparent that the area into which particles are
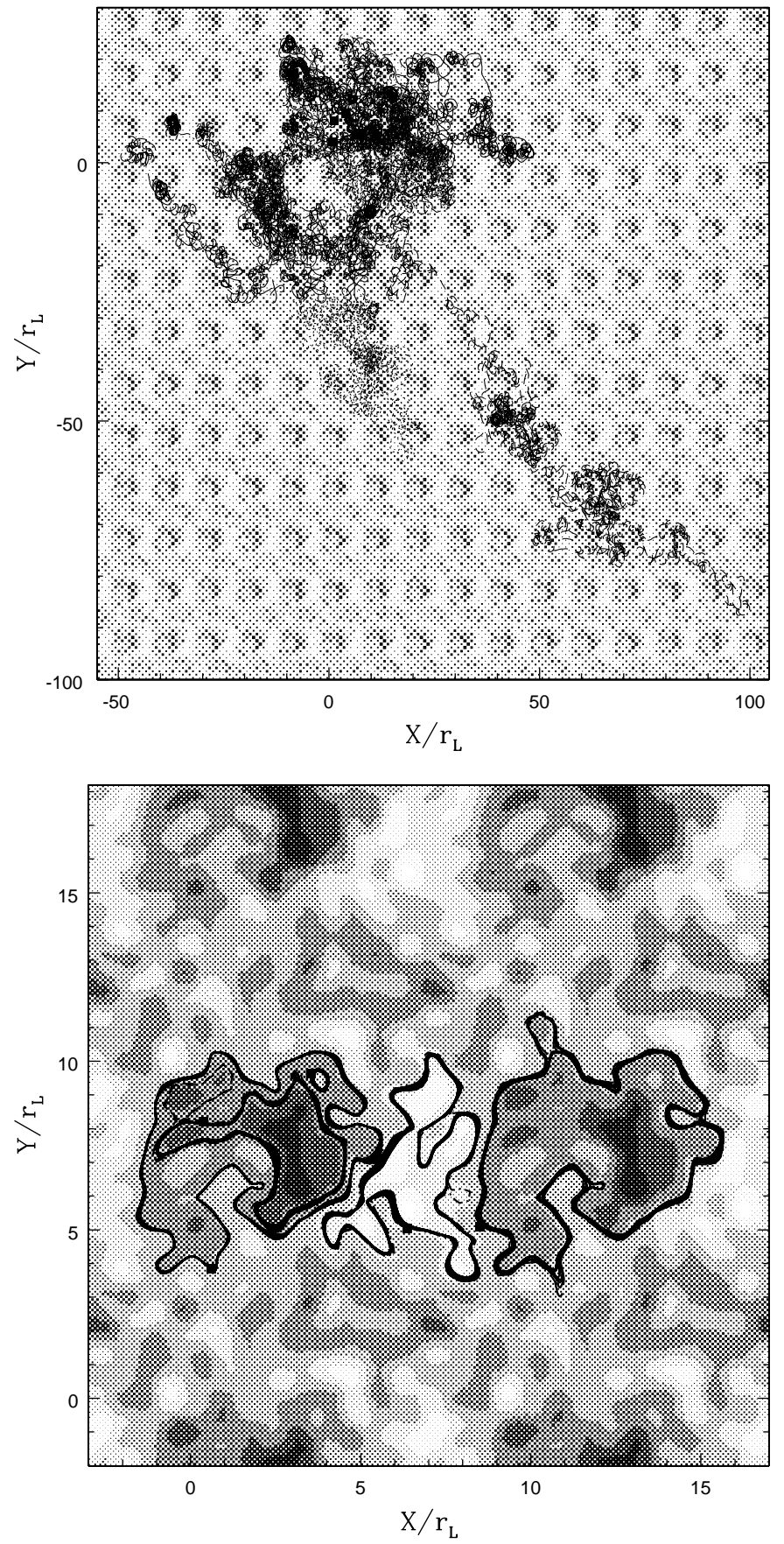

Fig. 2. Same as Fig. 1 but for $\lambda_{\mathrm{m}}=r_{\mathrm{L}}, \lambda_{\mathrm{M}}=10 r_{\mathrm{L}}$. Here the bottom panel refers to a tiny area of the plot on top.

contained grows with time much more effectively in the second case indicating that the wavelengths appropriate to enhance diffusion are those comparable with the particle Larmor radii.

This is made quantitative by Fig. 3 in which we plot the squared displacement averaged over the ensemble of particles for a number of different simulations, each relating to a particular magnetic field configuration. The curves marked with empty triangles in the first two panels, from top to bottom, refer to the average behaviour of particles in the field configurations that are shown in Figs. 1 and 2 respectively. It is apparent that particles travel far from their initial positions much 

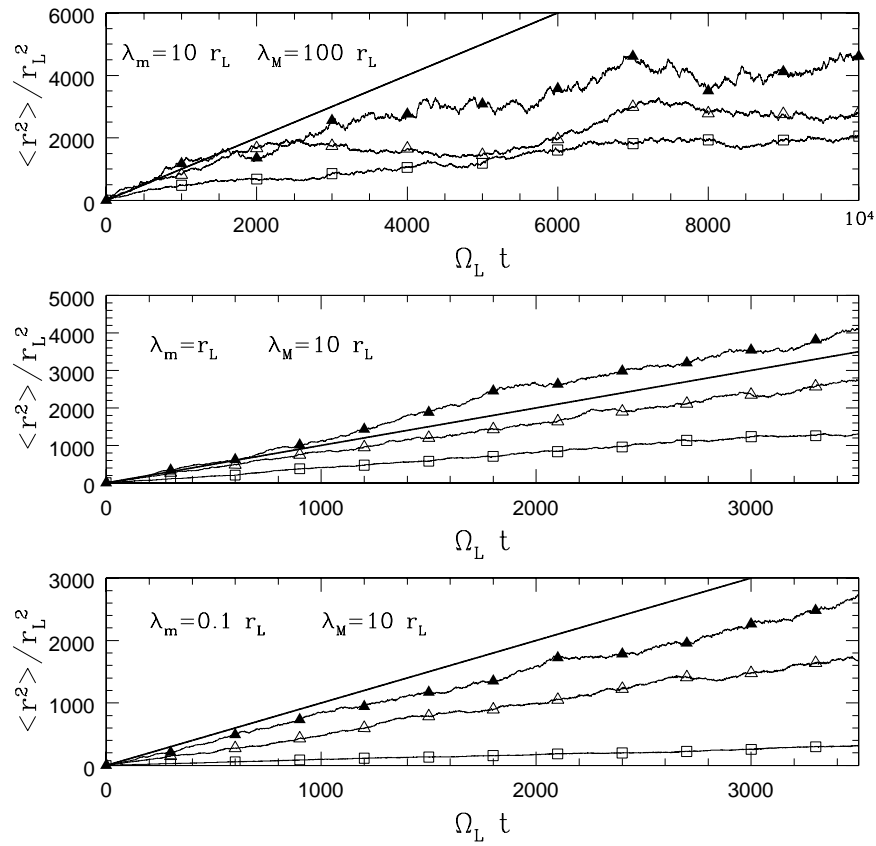

Fig. 3. Average squared displacement as a function of time. The curves marked with triangles refer to a perturbation spectrum $\propto k^{-1}$ (empty triangles correspond to a perturbation amplitude $\delta B / B_{0}=.5$ while the filled ones correspond to $\delta B / B_{0}=1$ ), while the curves marked with squares refer to the flat spectrum $\left(k^{0}\right)$ case. The vertical panels refer, from top to bottom to the case of a perturbation spectrum extending from $10 r_{\mathrm{L}}$ to $100 r_{\mathrm{L}}$ (top panel), from $r_{\mathrm{L}}$ to $10 r_{\mathrm{L}}$ (middle panel) and from $0.1 r_{\mathrm{L}}$ to $10 r_{\mathrm{L}}$ (bottom panel) respectively. In all panels the black solid line represents Bohm diffusion.

more efficiently when they experience perturbations of the field within a gyration period. Nonetheless, perturbation scales that are smaller than the particle gyration radius are less effective than "resonant ones". In fact, when one compares a flat spectrum $\left(k^{0}\right.$, curves marked with squares) with a $k^{-1}$ spectrum (curves marked with triangles), one always finds that the latter is more effective at producing particle diffusion, and the difference between the two increases in the three panels from top to bottom. In the top panel the field spectrum does not make much of a difference for the particle behaviour, while in the third panel the difference is considerable, with particles going almost five times further when the spectrum is $k^{-1}$. This is because, taking the spectrum to be flat, for a given perturbation amplitude $\delta B$, there is a waste of power, the amount of power invested in the short wavelength modes being larger.

As we mentioned one can properly speak of a diffusive behaviour only in cases when $\left\langle r^{2}(t)\right\rangle \propto t$. This does not seem to be the case for the top panel of Fig. 3. Here $\left\langle r^{2}(t)\right\rangle$ seems to flatten progressively. In fact the perturbation scale is large enough for the drift approximation to be a good representation of the behaviour of a large number of particles. The trajectories of these particles are closed and their maximum possible size is of the order of the maximum wavelength of the perturbation.

The fact that there are a considerable number of closed particle orbits also means that there are a large number of sections of the system that are non-chaotic. This can be seen by plotting the Lyapunov exponent as a function of time (see Fig. 4).
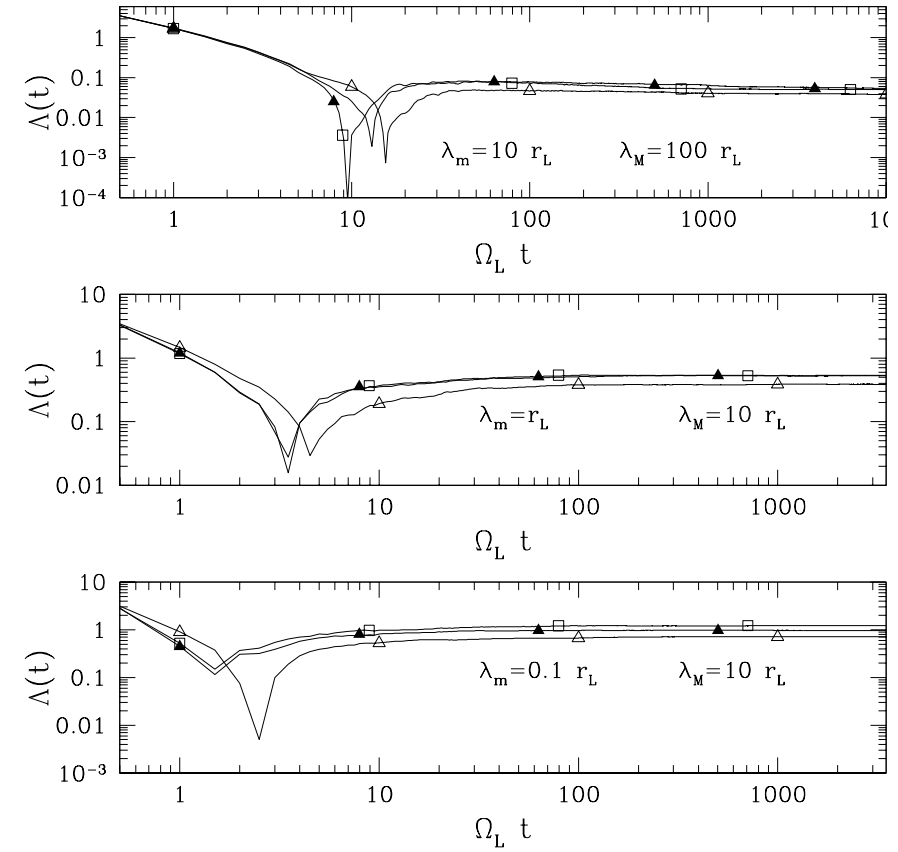

Fig. 4. Logarithmic plot of the Lyapunov exponent as a function of time. Same notation as in Fig. 3.

First of all it is clear from Fig. 4 that the origin of the diffusive behaviour is microscopic chaos. In fact the Lyapunov exponent is always positive for our system and tends to a constant value. However, at variance with the diffusion coefficient, this value is largest for perturbations with the largest power invested in short wavelength modes and decreases when one takes the field to vary mostly on large scales. In other words, although microscopic chaos is the source of particles diffusion, it is not possible to directly relate the efficiency of diffusion to the degree of chaoticity of the system, but there is no contradiction in this.

\section{A statistical description of particle diffusion}

In the following we give an approximate statistical treatment of the problem described by Eqs. (3), (1) aiming at an analytic estimate of the diffusion coefficient.

In principle, when the minimum length-scale over which the magnetic field varies is much larger than the gyration radius of the particles, we expect that the $\boldsymbol{\nabla} B$ drift (Eq. (13)) provides a reasonable description of the average particle motion. In this case the analytic computation of the diffusion coefficient is similar to what has been developed in a different context (Pettini et al. 1988, and references therein) along the line of the classical works of Dupree (Dupree 1967) and Taylor and McNamara (Taylor \& McNamara 1971). However, the motions described by Eq. (13) are not chaotic, no matter how complex the structure of the perturbing magnetic field is. The guiding center drift is, in fact, described by a set of just two independent ordinary differential equations. This prevents dynamical chaos from setting in, as the minimum number of independent first order differential equations required for this to happen is three (Guckenheimer \& Holmes 1983). 
Thus, in passing from Eqs. (3) to the vector Eq. (13), there is an important loss of information about dynamics. However, this is an artifact of the drift approximation which does not affect the computation of the velocity autocorrelation function (19) which, otherwise, should be computed using the equations of motion (3) and thereby eliminating the contribution of the fast gyration. At variance, in order to simplify the computations, one a priori eliminates the fast gyration by directly going to the drift approximation, then the loss of the source of diffusion - that is chaotic dynamics - is repaired by the legitimate assumption that $\boldsymbol{x}(t)$ in Eq. (19) is a random process.

The basic equation of motion (13) is rewritten as

$\boldsymbol{v}(t)=\frac{\mathrm{d}}{\mathrm{d} t} \boldsymbol{x}(t)=\boldsymbol{b}[\boldsymbol{x}(t)] \times \hat{\boldsymbol{k}}$

with $\boldsymbol{b}[\boldsymbol{x}]=\left(r_{\mathrm{L}} c / 2 B_{0}\right) \boldsymbol{\nabla} B_{1}[\boldsymbol{x}]$, where $B_{1}$ is the perturbing term in Eq. (1).

Let us consider the displacement $\boldsymbol{r}(t)=\boldsymbol{x}(t)-\boldsymbol{x}(0)$ of a single particle trajectory and introduce the mean square displacement $\left\langle r^{2}(t)\right\rangle$ averaged over an ensemble of trajectories. From the formal solution $\boldsymbol{x}(t)=\boldsymbol{x}(0)+\int_{0}^{t} \mathrm{~d} t^{\prime} \boldsymbol{v}\left(t^{\prime}\right)$ of Eq. (15), one can derive the diffusion equation

$$
\frac{\mathrm{d}}{\mathrm{d} t}\left\langle r^{2}(t)\right\rangle=2\langle\boldsymbol{v}(t) \cdot \boldsymbol{r}(t)\rangle=2 \int_{0}^{t} \mathrm{~d} \tau\langle\boldsymbol{v}(t) \cdot \boldsymbol{v}(t-\tau)\rangle
$$

The term in $\boldsymbol{x}(0)$ vanishes if the system is statistically invariant under spatial translations. For a standard Einstein-Fick diffusion process one has $\left\langle r^{2}(t)\right\rangle=D t$, with $D$ a constant (diffusion coefficient), so that by definition $\frac{\mathrm{d}}{\mathrm{d} t}\left\langle r^{2}(t)\right\rangle=D$ and by comparing with Eq. (16) we obtain the Green-Kubo expression

$D=2 \int_{0}^{\infty} \mathrm{d} \tau\langle\boldsymbol{v}(t) \cdot \boldsymbol{v}(t-\tau)\rangle$

for the asymptotic diffusion coefficient.

The Lagrangian velocity autocorrelation function, i.e. computed along a particle trajectory, is expressed as follows

$$
\begin{aligned}
\langle\boldsymbol{v}(t) \cdot \boldsymbol{v}(0)\rangle & =\langle\{\boldsymbol{b}[\boldsymbol{x}(t)] \times \hat{\boldsymbol{k}}\} \cdot\{\boldsymbol{b}[\boldsymbol{x}(0)] \times \hat{\boldsymbol{k}}\}\rangle \\
& =\left\langle-\hat{\boldsymbol{k}} \times \sum_{\boldsymbol{k}_{1}} C_{\boldsymbol{k}_{1}}(t) \mathrm{e}^{\mathrm{i} \boldsymbol{k}_{1} \cdot \boldsymbol{x}(t)} \cdot \sum_{\boldsymbol{k}_{2}} C_{\boldsymbol{k}_{2}}(0) \mathrm{e}^{\mathrm{i} \boldsymbol{k}_{2} \cdot \boldsymbol{x}(0)} \times \hat{\boldsymbol{k}}\right\rangle
\end{aligned}
$$

where we have assumed a time translational invariance of the autocorrelation function, and we have expanded $\boldsymbol{b}[\boldsymbol{x}(t)]$ in Fourier series. In the case of statistically homogeneous spatial fluctuations, the averaging $\langle\cdot\rangle$ over the initial condition $\boldsymbol{x}(0)$ is performed through an integration $\int \mathrm{d} \boldsymbol{x}(0)$ which yields a delta function $\delta_{\boldsymbol{k}_{1},-\boldsymbol{k}_{2}}$ thus simplifying Eq. (19) to

$$
\langle\boldsymbol{v}(t) \cdot \boldsymbol{v}(0)\rangle=\left\langle\sum_{\boldsymbol{k}} C_{\boldsymbol{k}}^{\perp}(t) C_{-\boldsymbol{k}}^{\perp}(0) \mathrm{e}^{\mathrm{i} \boldsymbol{k} \cdot[\boldsymbol{x}(t)-\boldsymbol{x}(0)]}\right\rangle,
$$

where $C_{\boldsymbol{k}}^{\perp}$ are the Fourier components of the magnetic field gradient in the plane perpendicular to $\hat{\boldsymbol{k}}$.

In order to make feasible any analytic estimate of the velocity autocorrelation function, two approximations are introduced: $i$ ) the average in Eq. (19) is split into the product of the average $\left\langle C_{k}^{\perp}(t) C_{-k}^{\perp}(0)\right\rangle$ and of the average of the exponential, this is reasonable if the particle motions are sufficiently complicated (chaotic) to destroy almost any correlation with the magnetic field structure; ii) $\boldsymbol{r}(t)=\boldsymbol{x}(t)-\boldsymbol{x}(0)$ varies in time as a random Gaussian process, in this case, assuming also an isotropic diffusion so that $\langle\boldsymbol{r}(t)\rangle=0$, the cumulant expansion of the average of the exponential gives $\left\langle\mathrm{e}^{\mathrm{i} k \cdot r(t)}\right\rangle=\mathrm{e}^{-\frac{1}{4} k^{2}\left\langle r^{2}(t)\right\rangle}$. Now, by deriving with respect to time Eq. (16) and using these two simplifying hypotheses in Eq. (19), we finally get

$$
\begin{aligned}
\frac{\mathrm{d}^{2}}{\mathrm{~d} t^{2}}\left\langle r^{2}(t)\right\rangle & =2\langle\boldsymbol{v}(t) \cdot \boldsymbol{v}(0)\rangle \\
& =2 \sum_{\boldsymbol{k} \in \Omega}\left\langle C_{\boldsymbol{k}}^{\perp}(t) C_{-\boldsymbol{k}}^{\perp}(0)\right\rangle \mathrm{e}^{-\frac{1}{4} k^{2}\left\langle r^{2}(t)\right\rangle},
\end{aligned}
$$

which is a nonlinear diffusion equation now in closed form. Note that if we assume a time $\delta$-correlation of the $C_{k}^{\perp}(t)$, which will be true with a suitable coarse graining of time, then $\left\langle C_{k}^{\perp}(t) C_{-k}^{\perp}(0)\right\rangle$ is replaced by $\left|C_{k}^{\perp}\right|^{2}$. Analytic - when possible - or numeric integration of Eq. (20) gives $\left\langle r^{2}(t)\right\rangle$ and hence $D=\lim _{t \rightarrow \infty} \frac{1}{t}\left\langle r^{2}(t)\right\rangle$.

In the model situation we described in the previous section, we may write Eq. (20) as

$\frac{\mathrm{d}^{2}}{\mathrm{~d} t^{2}}\left\langle r^{2}(t)\right\rangle=2 \frac{c^{2} r_{\mathrm{L}}^{2}}{4 B_{0}^{2}} \sum_{k \in \Omega} k^{2}\left|B_{k}\right|^{2} \mathrm{e}^{-\frac{1}{4} k^{2}\left\langle r^{2}(t)\right\rangle}$,

where $B_{k}$ are the Fourier components of the inhomogeneous part of the magnetic field. We assume that the wave vectors fill a 2-dimensional ring-shaped domain starting at radius $k$ and ending at $\rho k$, with $\rho \geq 1$, and further normalize the fluctuations as follows:

$(\delta B)^{2}=\sum_{k \in \Omega}\left|B_{k}\right|^{2} \simeq 2 \pi \int_{k}^{\rho k} \mathrm{~d} k k\left|B_{k}\right|^{2}$.

We adopt as before a spectrum with equal power at all modes $\left|B_{k}\right|^{2} \sim k^{-2}$, and a flat spectrum $\left(\partial B_{k} / \partial k\right)=0$.

Let us consider the former first. With the replacement

$\sum_{k \in \Omega} \rightarrow 2 \pi \int_{k}^{\rho k} \mathrm{~d} k k$

and the definitions

$\Gamma=k^{2}\left\langle r^{2}\right\rangle, \quad \theta=2 \frac{\delta B}{B} r_{\mathrm{L}} c k^{2} t$,

Eq. (21) may be rewritten as

$\frac{\mathrm{d}^{2} \Gamma}{\mathrm{d} \theta^{2}}=\frac{\mathrm{e}^{-\Gamma / 4}}{4 \Gamma} \frac{1-\mathrm{e}^{\left(1-\rho^{2}\right) \Gamma / 4}}{\ln \rho}$.

The limit $\rho \rightarrow 1$ admits a completely analytical solution, in fact Eq. (24) reduces to

$\frac{\mathrm{d}^{2} \Gamma}{\mathrm{d} \theta^{2}}=\frac{\mathrm{e}^{-\Gamma / 4}}{8}$

and

$\frac{\mathrm{d} \Gamma}{\mathrm{d} \theta}=\left(1-\mathrm{e}^{-\Gamma / 4}\right)^{1 / 2}$

whence

$\Gamma=8 \ln \frac{1+\mathrm{e}^{\theta / 4}}{2 \mathrm{e}^{\theta / 8}}$. 


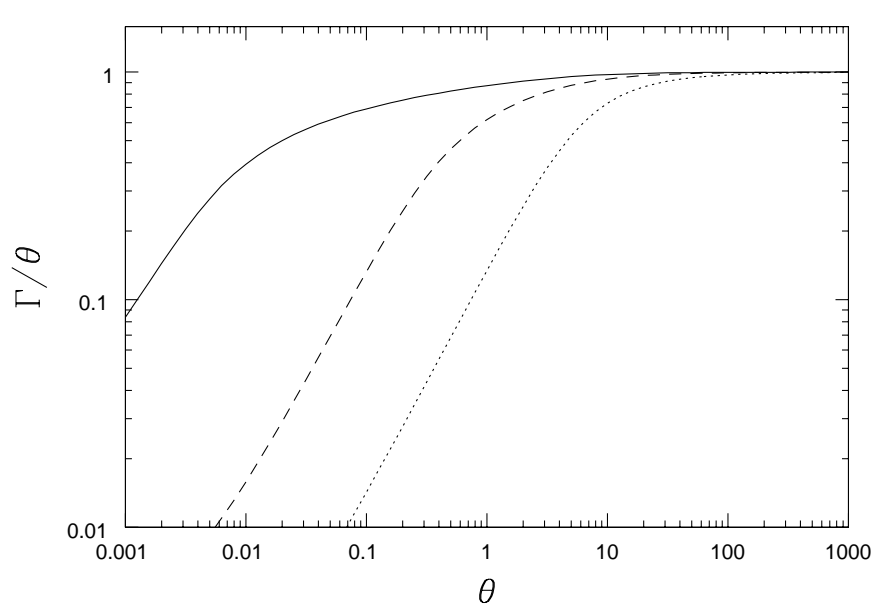

Fig. 5. Logarithmic plot of $\Gamma$ as a function of $\theta$ for $\rho=2,10,100$ : dotted, dashed and continuous curve respectively.

For values $\theta \ll 1$, that is at short times, we get

$\Gamma \simeq \frac{\theta^{2}}{16}$, i.e. $\left\langle r^{2}\right\rangle \simeq\left(\frac{\delta B}{B} \frac{r_{\mathrm{L}} c k}{2} t\right)^{2}$,

which is proper to a coherent motion occurring at the drift velocity if $\nabla B \simeq k \delta B$.

In the opposite limit of $\theta \gg 1$, thus at long times, we obtain, instead:

$\Gamma \simeq \theta$, i.e. $\left\langle r^{2}\right\rangle \simeq 2 \frac{\delta B}{B} r_{\mathrm{L}} c t$,

which is a Bohm type diffusion independent of $k$.

In general, that is for $\rho \gg 1$, numerical integration of Eq. (24) shows that there is always an initial regime for which $\Gamma \sim \theta^{2}$ and a long time asymptotic regime for which $\Gamma \sim \theta$, as it is apparent from Fig. 5.

With increasing extension of the spectrum, the asymptotic regime is reached in shorter time-scales, but the changes in the value of the diffusion coefficient are completely negligible. The conclusion one derives from this theoretical estimate is, therefore, that, within our modeling of the field, the Bohm estimate of the diffusion coefficient should still be an upper limit for cross-field motion of the particles. Bohm diffusion can be attained, but not largely overcome, for any value of the ratio $\delta B / B$ such that $\delta B$ can still be considered a perturbation.

Similar results are found when the flat spectrum is considered. With the same definitions of $\Gamma$ and $\theta$ as before, the relevant equation reads in this case:

$\frac{\mathrm{d}^{2} \Gamma}{\mathrm{d} \theta^{2}}=\frac{1}{2 \Gamma} \frac{1}{\rho^{2}-1}\left[\left(\frac{4}{\Gamma}+1\right) \mathrm{e}^{-\frac{\Gamma}{4}}-\left(\frac{4}{\Gamma}+\rho^{2}\right) \mathrm{e}^{-\frac{\rho^{2} \Gamma}{4}}\right]$,

so that by integrating once we obtain:

$\frac{\mathrm{d} \Gamma}{\mathrm{d} \theta}=\left[1-\frac{\mathrm{e}^{-\frac{\Gamma}{4}}}{\rho^{2}-1} \frac{4}{\Gamma}\left(1-\mathrm{e}^{-\frac{\rho^{2} \Gamma}{4}}\right)\right]$.

We integrated Eq. (31) numerically and found the result shown in Fig. 6 below. Also in this case the motion tends to be diffusive, after an initial phase during which the mean squared displacement grows more than linearly with time. Again the conclusion is that the value of the diffusion coefficient one should expect to observe is of order $D_{B}$.

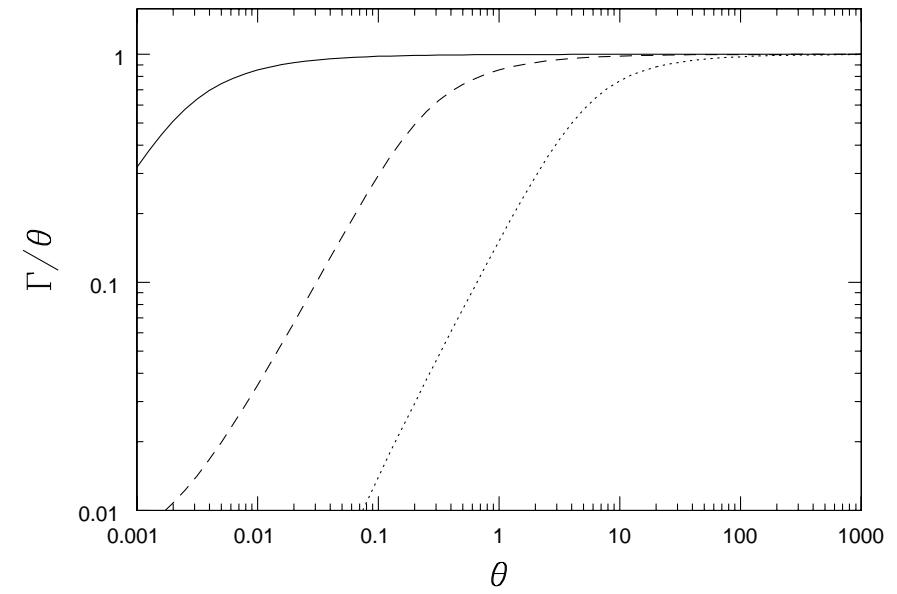

Fig. 6. Same as Fig. 5 but in the case of a flat spectrum $\left(B_{k} \propto k^{0}\right)$.
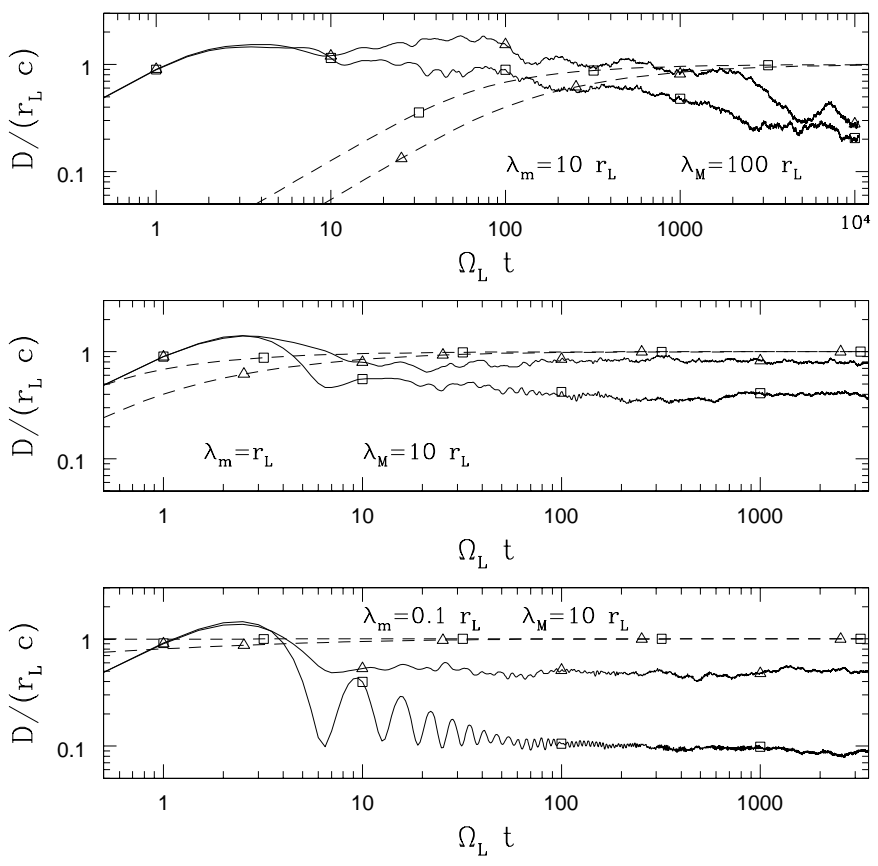

Fig. 7. Comparison between the theoretical and simulation behaviour of $\left\langle r^{2}(t)\right\rangle / t$ as a function of time for the case of a flat spectrum with $\delta B / B=0.5$ (curves marked with triangles) and for the case of a spectrum $\propto k^{-1}$ with the same value of $\delta B / B$ (curves marked with squares). Values on the vertical axis are in units of $r_{\mathrm{L}} c$, while the horizontal axis is in units of particle Larmor gyrations. The simulation results are shown as solid lines, while the statistical expectations are shown as dashed lines. For the different panels the caption is the same as in Fig. 3.

In Fig. 7 we compare the time-evolution of $\left\langle r^{2}(t)\right\rangle / t$ as found from our direct numerical simulations with that expected based on the statistical approach.

The first thing to notice is that in all cases we have considered, we have followed the particles' trajectories for a long enough time so that the asymptotic regime should have been reached, based on the statistical estimate. In fact, in the upper panel we cannot be sure that this is the case. The average squared displacement as a function of time is still decreasing when the simulation ends. Predicting what would happen if one 
followed the simulation for a longer time is not straightforward. The number of closed orbits, as we mentioned, is large in this case, but the fact that the Lyapunov exponent seems to tend to a positive value leaves open the possibility of a final diffusive behaviour. Of course, even if this is the case, the diffusion coefficient is going to be extremely small, and actually due to the very few particles whose orbits are not closed.

When the perturbation is on small scales, the behaviour is always diffusive, but with very different efficiencies depending on the details of the spectrum. The statistical approach is in all cases overestimating the value of $D$. It always predicts the maximum of efficiency, which we find in just one case, i.e. when most of the power in the perturbation of $\boldsymbol{B}$ is at wavelengths that are resonant with the particle gyro-radius.

\section{Conclusion}

In this paper we have pointed out a mechanism that provides a source of cross-field particle diffusion with minimal requirements on the degree of complexity of the magnetic field structure. We find that the intrinsic dynamical instability of the particle trajectories may lead to a diffusive behaviour even in the absence of time-dependence of the magnetic field or of 3-dimensional turbulence. The value of the diffusion coefficient is found to be of order $D_{B}$ when most of the perturbation power is invested in wavelengths that are of order of the particle Larmor radii.
Acknowledgements. Part of this work was supported by MIUR under grant Cofin-2001-02-10.

\section{References}

De Alcantara Bonfim, O. F., Griffiths, D. J., \& Hinkley, S. 2000, Int. Jour. Bifurc. Chaos, 10, 265

Benettin, G., Galgani, L., \& Strelcyn, J.-M. 1976, Phys. Rev. A, 14, 2338

Casse, F., Lemoine, M., \& Pelletier, G. 2001, Phys. Rev. D, 65, 023002

Dupree, T. H. 1967, Phys. Fluids, 10, 1049

Guckenheimer, J., \& Holmes, P. 1983, Nonlinear Oscillations, Dynamical Systems and Bifurcations of Vector Fields (NY: Springer-Verlag)

Jokipii, J. R., Kóta, J., \& Giacalone, J. 1993, Geophys. Res. Lett., 20, 1759

Jones, F. C., Jokipii, J. R., \& Baring, M. G. 1998, ApJ, 509, 238

Lichtenberg, A. J., \& Lieberman, M. A. 1992, Regular and Chaotic Dynamics (NY: Springer-Verlag)

Melrose, D. B. 1980, Plasma Astrophysics: Nonthermal Processes in diffuse magnetized plasmas, vol. II (Gordon \& Breach Science Publishers)

Ottaviani, M., \& Pettini, M. 1989, in Proc. of the Cadarache Workshop on Electrostatic Turbulence, EUR-CEA-FC-1381

Ottaviani, M., \& Pettini, M. 1991, Int. J. Mod. Phys. B, 5, 1243

Pettini, M., Vulpiani, A., Misguich, J., et al. 1988, Phys. Rev. A, 38, 344

Taylor, J. B., \& McNamara, B. 1971, Phys. Fluids, 14, 1492 